\title{
RNA topology
}

\author{
Maxim D. Frank-Kamenetskii
}

Department of Biomedical Engineering; Boston University; Boston, MA USA

Keywords: circular RNA, RNA interference, RNA topology, non-coding RNA, RNA

Submitted: 04/10/13

Accepted: 04/12/13

http://dx.doi.org/10.4161/adna.24680

Correspondence to: Maxim D. Frank-Kamenetskii; Email mfk@bu.edu
A new variety on non-coding RNA has been discovered by several groups: circular RNA (circRNA). This discovery raises intriguing questions about the possibility of the existence of knotted RNA molecules and the existence of a new class of enzymes changing RNA topology, RNA topoisomerases.

In the beginning of this year we witness an explosion of publications on a new form of noncoding RNAs (ncRNAs), circular RNA (circRNA). ${ }^{1-3}$ Although over decades researchers have occasionally reported on existence of circRNAs, these findings have been largely dismissed either as artifacts or, at best, as accidental errors of the splicing machinery. The three articles I am commenting on present a complete game changer: they convincingly demonstrate that circRNA is an abundant, evolutionary conserved form of ncRNAs. ${ }^{1-3}$ The functions of circRNAs are mostly a mystery although the data indicate that at least some of them serve as micro RNA (miRNA) sponges sequestrating miRNAs and thus attenuating the miRNAs gene silencing. ${ }^{3}$

What are the advantages for RNA to be in a circular from rather than in the traditional linear form? It looks like a major advantage is the enhanced stability in the intracellular environment full of exonucleases. However, as we have learned from many well studied cases, once evolution invents something new and advantageous, all kinds of unintended consequences emerge, which make it necessary for evolution to invent more and more mechanisms to cope with these unintended (and mostly undesired) consequences. In case of the RNA circularization, the immediate consequence is the possibility of knotting of separate circRNAs and the formation of topological links between different circRNA molecules. In other words, like discovery of circular DNA in the early 1960s eventually entailed an entire new field of DNA topology (see, e.g., ref. 4), the discovery of circRNAs must entail a new field of RNA topology.

We do not know whether knotting affects circRNA functioning but it is easy to imagine that linking must affect and thus must be avoided. In any case, by analogy with circular DNAs, one has to assume that RNA topoisomerases must exist, which deal with RNA topology. To the best of my knowledge, only one example of the RNA topoisomerase activity has been reported so far. ${ }^{5}$

It would be extremely interesting to figure out whether the newly discovered circRNAs could prove to be knotted. It is not a trivial task. It is not easy to distinguish between knotted and unknotted (trivial knots) single-stranded (ss) circular nucleic acids. These molecules are very flexible and form highly self-entangled coils so that differentiation between knots and unknots becomes a difficult task (see ref. 6). Perhaps the best way to study the issue is to convert ss circRNAs into the duplex form by the synthesis of the complementary strand (DNA or RNA). Duplex nucleic acids are much more rigid and distinguishing between knots and unknots can be done by EM, AFM or gel electrophoresis. As positive controls, one can use circRNAs subjected to RNA topoisomerase described by the Seeman's group. ${ }^{5}$ As was shown many years ago, subjecting of ss circDNA to a topoisomerase yields knotted molecules. ${ }^{6}$ 
Disclosure of Potential Conflicts of Interest

No potential conflicts of interest were disclosed.

\section{References}

1. Jeck WR, Sorrentino JA, Wang K, Slevin MK, Burd CE, Liu J, et al. Circular RNAs are abundant, conserved, and associated with ALU repeats. RNA 2013; 19:141-57; PMID:23249747; http://dx.doi. org/10.1261/rna.035667.112.
2. Memczak S, Jens M, Elefsinioti A, Torti F, Krueger J, Rybak A, et al. Circular RNAs are a large class of animal RNAs with regulatory potency. Nature 2013; 495:333-8; PMID:23446348; http://dx.doi. org/10.1038/nature11928.

3. Hansen TB, Jensen TI, Clausen BH, Bramsen JB, Finsen B, Damgaard CK, et al. Natural RNA circles function as efficient microRNA sponges. Nature 2013; 495:384-8; PMID:23446346; http://dx.doi. org/10.1038/nature11993.

4. Bates AD, Maxwell A. DNA topology. Oxford University Press, New York, 2005.
5. Wang H, Di Gate RJ, Seeman NC. An RNA topoisomerase. Proc Natl Acad Sci U S A 1996; 93:947782; PMID:8790355; http://dx.doi.org/10.1073/ pnas.93.18.9477.

6. Liu LF, Depew RE, Wang JC. Knotted singlestranded DNA rings: a novel topological isomer of circular single-stranded DNA formed by treatment with Escherichia coli omega protein. J Mol Biol 1976; 106:439-52; PMID:789893; http://dx.doi. org/10.1016/0022-2836(76)90095-4 